\title{
Economía politica de la extracción de recursos naturales: ¿nuevo modelo de desarrollo o imperialismo extractivo?
}

\author{
Henry Veltmeyer
}

Resumen. En este artículo se revisan los impactos económico, social y ambiental de la extracción de recursos naturales, así como sus implicaciones para el desarrollo y la política. El principal punto de atención es la dinámica del capital extractivo y el imperialismo del siglo xxi en la construcción del Estado posneoliberal en América Latina y la búsqueda de una forma de desarrollo más incluyente. También se explora el papel de Canadá en el imperialismo extractivista y en el proceso de desarrollo. Los gobiernos y diseñadores de políticas se han estancado en los extremos de un importante dilema: impulsar una estrategia de desarrollo con base en la explotación de fuerza de trabajo y la acumulación de capital humano o basarse en la inversión extranjera directa en la extracción ampliada de los abundantes recursos naturales de la región. Los conflictos derivados de la exportación del capital extractivo canadiense puede verse como el último giro y tendencia de una lucha de clase oculta en contra de las dinámicas del desarrollo capitalista. Palabras Clave: extractivismo, imperialismo, desarrollo, América Latina, Estado posneoliberal.

AвSTRACt. This article reviews the economic, social and environmental impacts of natural resource extraction, as well as its implications for development and policy. The main focus is on the dynamics of extractive capital and $21^{\text {st }}$ century imperialism in the construction of the post-neoliberal State in Latin America and the search for a more inclusive form of development. The role of Canada in extractive imperialism and in the development process is also explored. Governments and policymakers are stuck on the extremes of an important dilemma: promote a development strategy based on the exploitation of labor and the accumulation of human capital or depend on direct foreign investment and the widespread extraction of the region's abundant natural resources. The conflicts that stem from exporting Canadian extractive capital can be seen as the latest twist and a tendency in a class struggle against the dynamics of capitalist development.

KeywordS: extractive, imperialism, development, Latin America, post-neoliberal State.

Henry Veltmeyer es docente-investigador de la Unidad Académica de Estudios del Desarrollo de la Universidad Autónoma de Zacatecas, México. 


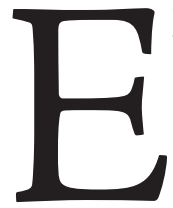

1 propósito de este artículo es reseñar los principales problemas que rodean a la profunda y creciente preocupación por los impactos económico, social y ambiental de la extracción de recursos naturales, así como sus implicaciones para el desarrollo y la política. El principal punto de atención de esta exploración sobre la economía política de la extracción de recursos naturales o, más precisamente, de la dinámica del capital extractivo y el imperialismo del siglo xxi, es el papel en la construcción de un Estado posneoliberal en América Latina y la búsqueda de una forma de desarrollo más incluyente. Un segundo punto de atención se enfoca en el papel que juega Canadá en este imperialismo extractivo y en el proceso de desarrollo.

Nuestro argumento está estructurado como sigue. En primer lugar, el artículo reseña el actual debate en relación con el proceso de desarrollo económico contemporáneo en América Latina y la búsqueda de un nuevo modelo de desarrollo. En este debate se incluye el cuestionamiento acerca de si la riqueza en recursos naturales constituye para un país una bendición o, como se ha argumentado a lo largo de los años, una maldición. ${ }^{1}$ Atendiendo a los desarrollos económico-políticos recientes en América Latina, el artículo argumenta que los gobiernos y diseñadores de políticas se han estancado en los extremos de un importante dilema: impulsar una estrategia de desarrollo con base en la explotación de la fuerza de trabajo y la acumulación de capital humano o basarse en la inversión extranjera directa en la extracción ampliada de los abundantes

/1/ La idea de una «maldición de recursos» es una ampliación de la idea de la enfermedad holandesa (dutch disease), acuñada originalmente por la revista The Economist en 1977 para hacer referencia a la manera en que el descubrimiento de petróleo, por parte de los holandeses en el mar del norte, precipitó su decadencia industrial. Mientras que la enfermedad holandesa centra la atención en las dinámicas de la tasa de intercambio de divisas, la tesis de la maldición de recursos, según fue establecida por Richard Auty (1993), enfatiza el papel del conflicto, la corrupción, la inestabilidad política y la volatilidad de los precios para explicar de qué manera los países que son ricos en recursos naturales no han logrado ascender en la escala del desarrollo. Aunque muchos de estos factores ayudan a explicar la maldición de los recursos, son meras manifestaciones de las dinámicas subyacentes del imperialismo y el capitalismo. 
recursos naturales de la región. Por supuesto, con frecuencia los diseñadores de política no se plantean esta alternativa, apuestan a explotar ambos recursos, humanos y naturales. En este punto, el artículo analiza brevemente las dinámicas regionales de extracción de recursos naturales bajo las condiciones de una transición histórica de un periodo de globalización neoliberal a una nueva era con fundamento en la dinámica de la política del nuevo extractivismo (Gudynas, 2010), al que hacemos referencia aquí como imperialismo extractivista. Se pone en cuestión un patrón de inversión extranjera directa dominada por el capital extractivo canadiense y las operaciones en América Latina de las compañías mineras con sede en Canadá y de propiedad canadiense que actúan con apoyo del Estado canadiense. Este artículo rastrea brevemente los impactos sociales, económicos y ambientales de estas inversiones y operaciones con objeto de resaltar las respuestas estratégicas de las comunidades afectadas o dañadas más directamente por aquéllas. En este punto, el artículo propone dos argumentos. Uno de ellos es que los conflictos derivados de la exportación del capital extractivo canadiense puede verse como el último giro y tendencia en una lucha de clase oculta en contra de las dinámicas del desarrollo capitalista. El segundo argumento es que el Estado canadiense es un importante protagonista en el nuevo imperialismo del siglo xxI.

\section{Para enmarcar el debate del desarrollo}

La extracción de recursos ha tenido un prolongado aunque controvertido papel en la historia del desarrollo capitalista. La intervención del Estado imperial en apoyo del capital extractivo - extracción de minerales, metales y otros recursos naturales no renovables, la inversión extranjera en la adquisición de tierra y agua (acaparamiento de tierra y de agua) — tienen una larga y tortuosa historia que puede rastrearse hasta la época mercantil del desarrollo capitalista (Girvan, 2012). A este respecto, es útil ubicar el imperialismo extractivo en una perspectiva histórica de «continuidad y cambio» en la evolución del 
capitalismo mundial y del imperialismo. De tal modo, puede recordarse que una de las motivaciones originales para la expansión imperialista europea hacia las Américas fue el deseo de metales preciosos. Las primeras instancias del imperialismo extractivo en las Américas fueron las minas de oro y plata en lo que actualmente es México y Sudamérica, que comenzaron a principios del siglo XVI. Más tarde, la necesidad de tierras adecuadas para el cultivo a gran escala de azúcar y otros productos tropicales y la explotación de la fuerza de trabajo esclava impulsó al imperialismo extractivo. Todavía más tarde, con la Revolución Industrial, el petróleo, el gas natural, el cobre y la bauxita ocuparon papeles centrales. El imperialismo extractivo ha adoptado, pues, diferentes formas a lo largo de la época de la conquista, el capitalismo comercial, el surgimiento del capitalismo industrial y el surgimiento del capitalismo monopólico hasta la época actual de las mega-corporaciones globales aliadas con el capital financiero.

El periodo de desarrollo capitalista posterior a la Segunda Guerra Mundial - que engloba la era del Estado desarrollista keynesiano y las subsecuentes elevación y caída del orden mundial neoliberal - significó un giro de retirada de la extracción de recursos como modelo económico hacia la explotación de lo que Arthur Lewis describiera como una «ilimitada reserva de fuerza de trabajo excedente» generada por el desarrollo capitalista de la agricultura, y lo que los marxistas siguen describiendo como «acumulación por despojo» (separando a los productores a pequeña escala, es decir, los campesinos, de sus medios de producción, obligándolos a abandonar la agricultura y asumir una de las vías de desarrollo para salir de la pobreza rural, el trabajo agrícola asalariado, las empresas agrícolas o la migración). ${ }^{2}$

12/ Varios autores han sugerido que las prácticas de la minería en América latina son típicas de lo que David Harvey (2003) denomina «acumulación por despojo». En las dinámicas contemporáneas de la «acumulación primitiva» (Marx) y la acumulación por despojo, véase Borras et al. (2012), Kay y Franco (2012) y Sosa y Zwarteveen (2012). En la formulación clásica de Marx, este proceso de desarrollo capitalista y de transformación social incluía a la agricultura (es decir, la conversión de los 
En el contexto del modelo promovido por los pioneros de la economía del desarrollo en los años cincuenta y sesenta, muchos países en el Sur global de esa época perseguían una estrategia de desarrollo de los recursos humanos basada en la explotación de la fuerza de trabajo más que en la extracción de recursos naturales. Sin embargo, varios países en la periferia del sistema fueron empujados hacia una división internacional del trabajo en el que se les obligó a exportar materias primas a cambio de bienes manufacturados en el Norte. Este desarrollo —o subdesarrollo, según algunos- derivó en la construcción de una teoría que explicaba el fracaso de tantos países en la periferia del sistema para entrar en una vía de desarrollo sustentable en términos de «dependencia» - dependencia para el desarrollo de sus fuerzas productivas en la extracción de materias primas para la exportación en forma de mercancía relativamente no procesada-. En esta teoría estaba en juego la paradoja de cuántos países ricos en recursos no lograron desarrollarse y pueden contarse entre los países más pobres del planeta en la actualidad, mientras cuántos países carentes de recursos, como Japón y Corea, tuvieron éxito para hacer la transición. Una explicación de este fracaso lo proveyó la Comisión Económica para América Latina y el Caribe (CEPAL) de la Organización de las Naciones Unidas (ONU) - el estructuralismo latinoamericano- y la «teoría de la dependencia», cuando ambas atribuyeron el subdesarrollo a una estructura de relaciones internacionales en la cual los beneficios del crecimiento económico se dirigían a los productores de bienes manufacturados en el centro del sistema, mientras que sujetaban a los países de la periferia a condiciones como el deterioro de los términos del comercio o la transferencia de los excedentes que inhiben o evitan el desarrollo de sus fuerzas productivas.

\footnotetext{
campesinos en un proletariado) e incluía a las reservas de tierra (enclosures), derivando en una lucha encubierta por la tierra. En estudios más recientes de este proceso, las reservas de los territorios comunes se amplían para incluir tanto al agua como los recursos subterráneos, derivando no sólo en el acaparamiento de tierras sino en el acaparamiento de aguas, así como en el acaparamiento de los principales recursos (Kay y Franco, 2012).
} 
Otros economistas de la tradición liberal han propuesto una explicación bastante diferente, atribuyendo el fracaso a una «maldición de recursos» (Auty, 1993, 2001; Haber y Menaldo, 2012; Sachs y Warner, 2001). Los factores descriptivos o explicativos que proponen estos economistas van desde el impacto negativo del desarrollo de los recursos naturales a la tasa de intercambio en otros sectores — la llamada «enfermedad holandesa», por ejemplo-, la volatilidad de los precios de las mercancías en el mercado mundial, la propensión hacia un ciclo de rápido incrementos y rápida decadencia (boom-bust cycle), la susceptibilidad a la corrupción, la excesiva confianza en la inversión extranjera directa, la estructura de producción de enclave con escasas vinculaciones hacia adelante o hacia atrás con otros sectores de la economía y una estructura social que inhibe el desarrollo más amplio de las fuerzas productivas de la sociedad y deriva en formas desiguales y exclusivas de crecimiento y en conflictos y guerras por los recursos. De tal modo, desde el estructuralismo latinoamericano y la escuela marxista de la dependencia hasta los economistas neoclásicos y neoliberales, hay acuerdo en que la extracción de recursos ofrece una vía bastante difícil hacia el desarrollo.

Aunque las reglas del orden capitalista neoliberal mundial debilitaron la capacidad de los gobiernos en los Estados periféricos para impulsar una política industrial y romper esta trampa, para evitar la maldición de recursos los gobiernos adoptaron políticas dirigidas a atraer la inversión extranjera a la producción industrial para la exportación de bienes manufacturados en vez de la extracción de recursos naturales. Sin embargo, los cambios en la economía mundial en años recientes han dado origen a la noción de que esta vez será diferente, que las modificadas condiciones del mercado mundial permitirían ahora que los países ricos en recursos exploten su ventaja comparativa en recursos naturales y convertir lo que alguna vez fue una maldición en una bendición. Los exponentes de esta perspectiva incluyen a varios economistas del Banco Mundial, quienes han publicado una serie de estudios que sugiere que el aumento reciente en la inversión extranjera a gran escala en tierra y en la extracción y explotación de los recursos naturales bajo las actuales 
condiciones tiene implicaciones positivas para muchos países en desarrollo, permitiéndoles aprovechar las nuevas oportunidades que ofrece una fuerte demanda de estos recursos en el mercado mundial (Collier y Venables, 2011; Deininger y Byerlee, 2011; Banco Mundial, 2010, 2011). Lo que se requiere, dicen estos economistas, es un enfoque de manejo sustentable de los extraíbles y una administración efectiva de los conflictos socioambientales inevitables (Bebbington et al., 2009; Bebbington, 2011).

No todos están de acuerdo con esta evaluación positiva de la bendición de los recursos y la visión de la industria extractiva como algo saludable y la relación entre esta industria y los guardianes del patrimonio mundial de recursos naturales, en especial de los pueblos indígenas. En efecto, algunos críticos han sugerido, desde una perspectiva de la economía política, que la extracción de recursos naturales es una forma particularmente atrasada del capitalismo con una diversidad de resultados perversos y que los costos asociados exceden con mucho cualesquiera beneficios potenciales (Gudynas, 2011a; 2011b). El argumento de estos críticos es que la extracción de recursos naturales es particularmente propicia a las condiciones de desarrollo desigual (inequidades sociales en la distribución de riqueza e ingreso), en donde los beneficios potenciales del crecimiento económico son superados por los costos económicos y socioambientales asociados, derivando en condiciones de inestabilidad por el conflicto político e incluso guerras por los recursos (Bannon y Collier, 2003; Collier, 2003).

De tal modo, al debate teórico se unieron quienes proponían y quienes se oponían a la extracción de recursos naturales y la exportación de mercancías primarias como una estrategia de desarrollo. Pero a nivel de la práctica del desarrollo y de las políticas no hay debate. En América Latina, el rápido aumento de las mercancías primarias en el mercado mundial señaló un giro hacia lo que se ha llamado el nuevo extractivismo, un apoyo a la inversión extranjera directa en el sector de los recursos naturales y en la exportación de recursos naturales en forma de mercancías primarias. Lo que resulta novedoso de este cambio estratégico es que está basado en un nuevo régimen de políticas diseñado para asegurar una división más equitativa de la riqueza de los recursos 
mediante el cobro de rentas por el gobierno, o que los recursos mismos, en algunos casos - Bolivia, por ejemplo—, son nacionalizados y administrados de manera sustentable. En la práctica, esto significa un nuevo régimen regulatorio diseñado para regular la inversión extranjera en la extracción de recursos y la operación de las compañías mineras, con objeto de asegurar un mejor resultado en el desarrollo económico y para mitigar o proteger a la sociedad y la economía, así como al ambiente, de las extremadamente bien documentadas consecuencias negativas del desarrollo de los recursos naturales.

\section{El nUeVo extractivismo:}

RETORNO AL SIGLO XIX

El súbito aumento en las mercancías primarias ha traído consigo un sinfín de cambios en el paisaje del desarrollo nacional. Los países exportadores de mercancías - especialmente en Sudamérica, en donde los gobiernos han adoptado la extracción de recursos naturales como estrategia de desarrollo- se han beneficiado sustancialmente de los ingresos derivados de la exportación, con rápido crecimiento y una economía subyacente boyante. Las tasas anuales de crecimiento han fluctuado entre 4.5 y 7.2\% (5\% en promedio) de 2002 a 2008 (CEPAL, 2009). ${ }^{3} \mathrm{Al}$ mismo tiempo, tras un largo periodo de limitado desempeño, la inversión en el sector de la extracción de recursos y de las mercancías primarias ha renacido y en años recientes se ha acelerado (Cypher, 2010). En

13/ Con los términos comerciales elevándose en $22.8 \%$, el ingreso real per cápita en América latina se elevó en promedio en 21\% en el periodo 2003-2008 (CEPAL 2009, tablas A-1 y A-12). Desde principios de 2008, los precios de las mercancías se derrumbaron aceleradamente hasta principios de 2009. Para abril de 2010, siguiendo el subíndice de las materias primas para la industria de la oficina de investigación de mercancías (Commodity Research Bureau's Raw Industrials Sub-Index) de 13 mercancías, los precios de las mercancías se habían recuperado notablemente - estaban apenas 6\% por debajo de su punto alto en 2008, pero más del doble de sus niveles para 2003 (Cypher 2010, 566). 
los últimos veinte años, Sudamérica ha sido objeto de grandes inversiones del capital extractivo internacional. Según el Banco Mundial (2005: 20), en el periodo 1990-1997 la inversión mundial en la exploración minera aumentó 90\%. Sin embargo, para América Latina, este crecimiento fue de $400 \%$ y para Perú de 2000\% (Lust, 2012). En 1997, América Latina atrajo 40\% de la inversión en minería (De Echave, 2008: 21), mientras que en 2010 América Latina fue la cuarta en inversiones a nivel mundial en exploración minera (Panfichi y Coronel, 2011:395).

América Latina ha experimentado un fuerte crecimiento económico alrededor de 2003, en particular durante el periodo que derivó en la llamada crisis financiera global, a un ritmo que no se había visto desde los años setenta. ${ }^{4}$ Un súbito incremento en las mercancías, que se manifestó en Sudamérica en particular, ha constituido el motor detrás de este crecimiento. $\mathrm{Al}$ mismo tiempo, aunque los términos del comercio se han trasladado fuertemente a favor de materias primas suaves como soya, trigo, maíz y caña de azúcar, se han hecho mucho más favorables para las materias primas duras como el cobre, el estaño y el petróleo (Cypher, 2010).

Iniciada bajo condiciones de una crisis económica, la primera década del nuevo milenio en América Latina vio cómo una oleada de regímenes popu-

14/ En varios reportes sobre América Latina producidos por la CEPAL desde 2007, el actual periodo de crecimiento se ha etiquetado como extraordinario, perfilándose como la expansión más prolongada y más fuerte durante un buen tiempo, permitiendo que el ingreso per cápita en la región se elevara en un promedio de $20 \%$ de 2003 a 2008, y una cifra cercana en los seis años siguientes. Sin embargo, la CEPAL ha sido menos entusiasta en cuanto al resultado probable de este rápido crecimiento, es decir, acerca de si podría conducir a la transformación productiva y social que se necesitaría para sustentar un proceso de desarrollo económico y social. El principal obstáculo en esta instancia es el constreñimiento estructural representado por las excesivas inequidades en el acceso a recursos productivos, riqueza e ingreso y oportunidades para la autoproyección. En otras palabras, lo que se necesitaría pero que no se encuentra (ni siquiera en los regímenes post-neoliberales «progresistas» de centroizquierda) es un ataque concertado en la estructura de la (excesiva) inequidad y una mayor inversión de los ingresos generados en el sector de la extracción en infraestructura, innovación tecnológica y desarrollo de recursos humanos (CEPAL, 2010). 
listas «rojos» (o «rosas») de centro-izquierda asumía el poder, a horcajadas sobre la ola de sentimiento antineoliberal (Lievesley y Ludlam, 2009; Petras y Veltmeyer, 2005). El significado de este cambio de régimen es muy controvertido, como lo es el giro hacia la extracción de recursos, en particular en los regímenes progresistas posneoliberales y el súbito incremento de mercancías con que coincidió. La visión positiva es que una estrategia de extracción de recursos ofrece los ingresos fiscales necesarios para financiar una forma socialmente más incluyente, equitativa y justa de desarrollo basado en un consenso post-Washington — primero, reducir la pobreza y, segundo, ampliar una clase media de individuos con poder adquisitivo (Infante y Sunkel, 2009). ${ }^{5}$

El significado e implicaciones de este desarrollo así entendido también han sido muy debatidos. Como se ha resaltado antes, algunos ven en él perspectivas para un desarrollo incluyente (nuevo desarrollismo) financiado por la exportación de mercancías primarias. Otros son escépticos. En primer lugar, los escépticos argumentan que, a pesar del éxito que han tenido algunos países para reducir la pobreza, hay escasas evidencias de un compromiso de estos regímenes posneoliberales con el cambio social sustantivo que se requiere para tratar la inequidad persistente y, además, el apoyo de estos regímenes en la Inversión Extranjera Directa (IED) para la extracción de recursos naturales tiene implicaciones desarrollistas y políticas negativas. En cuanto a la suposición de que los regímenes posneoliberales canalizarían los ingresos fiscales adicionales derivados de la renta de recursos y de la fiscalización de las exportaciones de materias primas hacia programas sociales incluyentes y asignarían una mayor porción de los ingresos fiscales en dirección a la igualdad social (para cerrar

15/ En cuanto al papel del Estado en esta estrategia post-neoliberal — bautizada como «activismo incluyente de Estado» por Arbix y Martin (2010) y un rasgo del nuevo desarrollismo (Bresser-Pereira, 2007 y 2009) - se basa en la idea de que más que constituir una maldición, la explotación de recursos como los minerales y los hidrocarburos o combustibles fósiles genera rentas fáciles de ser fiscalizables que pueden financiar el desarrollo social (Stijns, 2006). 
los huecos existentes en ingreso y riqueza), hay escasa evidencia de que eso haya ocurrido, con excepción de Venezuela, en el régimen de Hugo Chávez.

En pocas palabras, los trabajadores, un importante elemento del sector popular, no se han beneficiado de la oleada de actividades y desarrollos relacionados con la minería. Por ejemplo, en el caso nada atípico de las compañías mineras en Argentina y Chile, los salarios pagados se estiman en sólo 6\% del valor actual de las exportaciones mineras (Solanas, 2007aः 2). Además, a pesar del súbito crecimiento en las mercancías, los trabajadores han recibido poco en términos de aumentos de sueldos. Un índice de los sueldos promedio reales en el sector formal del mercado de trabajo en Argentina, Bolivia, Brasil, Chile, Colombia, México, Perú, Uruguay y Venezuela muestra algunos resultados desalentadores. Usando el 2000 como año base, los datos de la CePAl señalan un aumento acumulado en los sueldos promedio de únicamente $0.46 \%$ para 2006 (CEPAL, 2007: tabla A-28). Cypher (2010: 588) concluye que los «trabajadores nada han recibido» del súbito incremento de las mercancías. Los estudios realizados por Petras y Veltmeyer (2009) en Brasil, así como en Bolivia y Ecuador, apuntan hacia resultados igualmente desalentadores. A pesar de la reducción en la incidencia de la pobreza entre quienes reciben ingresos (de $40 \%$ a 20\% en estos países de 2003 a 2008), y la inclusión de quienes tienen escasos ingresos en los programas sociales de los gobiernos (salud, educación y bienestar mínimo), hasta la fecha existe evidencia de escasa a nula de mejoramiento en la condición social de las personas en el sector popular de la sociedad, es decir, los trabajadores rurales sin tierras o semiproletarizados y los campesinos en pequeña escala y el proletariado urbano de los trabajadores informales (Veltmeyer y Tetreault, 2012).

Uno de los más destacados hechos acerca del nuevo extractivismo consiste en que atraviesa los tipos de regímenes. Es posible ubicar a los regímenes de política de América Latina en tres categorías: 1) neoliberal (Colombia, México); 2) «tercera vía» posneoliberal (Argentina, Brasil, Chile), y 3) populista radical y nacionalista en recursos o protosocialista (Bolivia, Ecuador, Venezuela). Los estudios recientes acerca de las dinámicas de las políticas del nuevo extracti- 
vismo muestran un apoyo equitativo en la extracción de recursos naturales, la inversión extranjera directa y la exportación de mercancías primarias entre los tipos de régimen. Aunque los regímenes neoliberales de política son a todas luces menos reguladores y más amigables con la inversión extranjera en busca de recursos, los inversionistas extranjeros y el capital extractivo no parecen preferir un tipo específico de régimen. Sin duda, esto se debe a que los regímenes posneoliberales o progresistas (socialmente incluyentes) como los de Argentina, Brasil y Chile están limitados por el hecho de que las actividades extractivas están altamente localizadas y el capital tienda a fluir hacia los enclaves en donde los recursos están localizados, en donde las inversiones extranjeras son bienvenidas y legalmente seguras y en donde, por ende, se anticipan altos rendimientos de las inversiones. Pero lo que es significativo acerca de la inversión extranjera «en busca de recursos» en la región es que parece estar igualmente en casa y ser bien recibida en los regímenes posneoliberales y en los que son nacionalistas en cuanto a los recursos al igual que en los regímenes que abiertamente son noregulatorios (neoliberales) como Colombia y México. La razón para que esto suceda no es difícil de encontrar: la confianza en la inversión extranjera para la explotación y el desarrollo de las reservas de recursos naturales del país y una coincidencia fundamental de los intereses económicos entre las corporaciones mineras multinacionales y los gobiernos en la región. No es únicamente un accidente geográfico sino también una coincidencia de intereses.

\section{InVERsión CANADIENSE EN AmériCA LATINA}

La primera oleada de flujos de capital hacia América Latina bajo la guisa de IED se dio entre 1990 y 1994, bajo condiciones generadas por la imposición de la agenda de reforma de las políticas del consenso de Washington - en particular, la liberalización, desregulación y privatización-. Al inicio del siglo XxI, bajo condiciones bastante diferentes (un giro en la ola en contra de la globalización neoliberal), hubo otra oleada de IED, pero esta vez más 
centrada en los recursos naturales («IED en busca de recursos»), con el capital canadiense detentando el papel principal (Arellano, 2010). ${ }^{6} \mathrm{El}$ capital canadiense fue especialmente agresivo en sus adquisiciones y operaciones en el sector minero. Para fines de la primera década del nuevo milenio, el capital canadiense equivalía a cerca de $40 \%$ de los proyectos globales de exploración minera, incluyendo 1,010 proyectos tan sólo en Sudamérica y 578 en México (véase InfoMine, 〈http://www.mining,ca〉).

Los estudios de CEPAL (2010) documentan el alcance y escala de la inversión extranjera en el sector de los recursos naturales en América Latina y el surgimiento de Canadá como una fuente importante de inversión directa en el subsector de la exploración mineral y la minería. En parte como consecuencia de la participación del capital canadiense, los flujos de llegada del IED hacia América Latina y el Caribe alcanzaron una cima sin precedentes en 2008. Exceptuando los centros bancarios en el Caribe, la región recibió 128.3 miles de millones de dólares en IED, superando en 13\% el nivel más alto alcanzado en 2007 (CEPAL, 2010).

El capital minero canadiense, a la cabeza de esta oleada de inversión, logró una posición dominante en la industria de la extracción, en particular en la minería de oro y otros metales preciosos. De hecho, representa un fenómeno global, y el capital canadiense ha llegado a dominar la industria en todo el planeta, al incluir importantes operaciones en África. Pero está particularmente bien posicionado en América Latina, en donde, con el apoyo del Estado canadiense, las compañías canadienses han establecido un monopolio virtual al llegar a dominar el sub-sector de la minería del oro y la plata. ${ }^{7}$ En México,

\footnotetext{
${ }^{16 /}$ En los años ochenta, la reserva de IED canadiense creció alrededor de 5\% a 10\% del PIB, pero en las siguientes dos décadas se elevó a cerca de 35\% del рів, excediendo eventualmente las reservas de llegada de IED. En la actualidad, Canadá es de hecho un inversionista neto en el extranjero, la mayor parte en relación con lo que Arellano (2010:2) denomina «IED en busca de recursos» (en contraposición de la IED que busca eficiencia).

${ }^{17 /}$ América Latina es el más importante destino para el capital minero canadiense, superando a África
} 
por ejemplo, se calcula (López y Eslava, 2011:28) que hasta 23\% del territorio nacional ha sido concesionado al capital extractivo para la exploración $y$ minería de minerales, y que $70 \%$ de estas concesiones para explorar y extraer minerales se han dado a firmas canadienses. En Argentina, un importante lugar en el mundo para el reciente acaparamiento de tierras por extranjeros, un mapa construido por «laangosturadigital maps» (Anónimo, 2012a) muestra la difusión y el grado del control extranjero del territorio nacional argentino. Según el mapa, casi 30 millones de hectáreas de la mejor tierra y de suelos fértiles, acuíferos y reservas naturales - y reservas de minerales estratégicosen 23 provincias ya son propiedad extranjera y otros 13 millones de hectáreas están actualmente a la venta. De tal manera, incluso en la era de la «ola rosa», América Latina ha cedido buena parte de su territorio para la exploración y buena parte del control de sus industrias extractivas ante las corporaciones transnacionales con sede en los centros imperiales. ${ }^{8}$

En 2009 las compañías mineras canadienses registradas en la bolsa de valores de Toronto Stock Exchange realizaron 1,010 proyectos en Sudamérica, 578 más en México, en comparación con 703 en África y 345 en Australia, Nueva Zelandia y Papúa Nueva Guinea. Muchos de estos proyectos están relacionados con la extracción de oro, plata, níquel y cobre en minas a cielo abierto. Las compañías con sede en Canadá representan 60\% de las inversiones extranjeras en el sector minero de la economía global (70\% en América Latina) y tienen cerca de $40 \%$ de los derechos globales de explotación mineral. ${ }^{9}$ En Co-

por un amplio margen. Más de la mitad de los recursos globales de las compañías mineras canadienses están ubicadas en América Latina y tienen un valor cercano a los 57 mil millones de dólares canadienses (Keenan 2010: 30, con base en información no publicada, Natural Resources Canada).

18/ Como se señaló en el caso de México, hasta el 23\%, del territorio nacional está concesionado a las compañías mineras para su exploración, perforación y extracción, pero en Colombia y Perú, en donde la industria extractiva domina la economía es aún mayor - $40 \%$ en el caso de Colombia y tanto como un $72 \%$ en el caso de caso de Perú.

19/ Según Díaz (2012), Toronto es la capital mundial de inversión minera, que representan 60\% de las empresas mineras registradas en los distintos mercados de valores y $80 \%$ de las transacciones financieras. 
lombia, lugar en el que el capital extractivo de origen canadiense ha estado muy activo en años recientes, sin duda por las condiciones particularmente favorables para la repatriación de las ganancias y el relativo éxito del actual gobierno para «pacificar» las regiones controladas por la guerrilla e iniciar negociaciones con las Fuerzas Armadas Revolucionarias de Colombia (farc), el 52\% de todas las inversiones son canadienses (Ministerio de Minas y Energía, 2012).

\section{El Estado Canadiense: ¿agente de desarrollo incluyente}

(UN NUEVO MODELO) O CAPITAL EXTRACTIVO?

Como ya hemos establecido, las compañías mineras canadienses son los principales jugadores en el envío global de capital en la economía política de la extracción de recursos. Sin embargo, el papel de Canadá de ninguna manera se restringe a la inversión en las operaciones de extracción de sus compañías mineras. El gobierno federal, como agencia del Estado canadiense bajo el régimen de Harper, también desempeña un importante papel para facilitar estas operaciones en apoyo del capital minero canadiense - un papel que puede describirse adecuadamente como «imperialista» (Engler, 2012) - . Un estudio reciente de la relación entre el Estado y el capital de origen canadiense respecto a la economía política de la extracción de recursos naturales la designa como «Canadá imperial» (Deneault y Sacher, 2012). Además, al menos para esta explicación, Canadá es el «santuario legal preferido para las industrias mineras mundiales». Dado su papel para asegurar la dominación de las multinacionales mineras con sede en Canadá, el Estado canadiense se describe mejor como un régimen imperialista extractivo («Imperial Canada, Inc.», como la llamarían Deneault y Sacher).

El papel activo del gobierno en apoyo del capital extractivo (las compañías mineras multinacionales de Canadá) ha asumido múltiples formas, incluyendo: 1) un cabildeo agresivo y apoyo diplomático de los esfuerzos de estas compañías por obtener concesiones para explorar y las licencias sociales para 
operar en los países anfitriones; 2) el uso de los considerables recursos del Departamento de Asuntos Extranjeros en apoyo diplomático e incluso financiero de las compañías mineras canadienses; ${ }^{10} 3$ ) asociarse con otros gobiernos en el diseño de un marco global de políticas mineras ${ }^{11} \mathrm{y}$, de hecho, escribir la legislación minera para algunos países; 4) patrocinar una serie de mesas redondas provinciales sobre la industria de extracción de recursos; y 5) un apoyo sustantivo para la agenda corporativa de responsabilidad social (CRS) de la ONU, que está diseñada explícitamente para que los gobiernos anfitriones garanticen licencias de operación en áreas social y ambientalmente sensibles y en terrenos altamente controvertidos. Además, y más significativamente, el programa canadiense de cooperación internacional, administrado por Canadian International Development Agency (CIDA), ha sido transformado en un agente de apoyo financiero para las compañías mineras canadienses en sus operaciones en el extranjero. El desvío de los fondos para la cooperación internacional administrados por el CIDA (ayuda exterior) para apoyar activamente a las compañías mineras canadienses - convirtiendo, efectivamente, sus operaciones en un proyecto de desarrollo - se ha justificado haciendo referencia a estos fondos y a estos proyectos como asistencia para los países involucrados para trasladarse hacia el «desarrollo incluyente». La teoría (más bien ideología) detrás de esta justificación —el uso de fondos públicos para la ganancia privada, como la ven

/10/ Este apoyo incluye representaciones y apoyo diplomático sobre los gobiernos anfitriones a nombre de las compañías mineras, crédito ampliado de parte de la Export Development Corporation a estas compañías, la construcción de sociedades públicas-privadas en el sector minero y asistencia internacional para el desarrollo - entrega de financiamiento para la cooperación internacional (CIDA) y apoyo para asistir a las compañías canadiense en sus negociaciones con los gobiernos y comunidades locales (para obtener concesiones para la explotación minera y licencia para operar). Sobre estos temas, véase Coumans (2012); Engler (2012) y Mining Watch (2012).

111/ Esta estrategia de sociedades ha adoptado la forma del Foro Intergubernamental sobre Minería, Metales y Desarrollo Sustentable (IFMMSD), una organización internacional apoyada por la oNU, con 45 países con abundantes minerales y metales, incluyendo a Canadá, uno de tan sólo dos países desarrollados. Otros incluyen a Rusia, la India y Sudáfrica, nueve países latinoamericanos y 23 de África subsahariana y Filipinas (uno de dos países asiáticos). 
Dawn Paley y Sakura Saunder (2012) — consiste en que el sector privado es la fuerza motriz en el proceso de generar crecimiento incluyente. Según un informe reciente del Comité de la Casa de los Comunes sobre asuntos extranjeros y desarrollo internacional (House of Commons, 2012), el papel del sector privado en el desarrollo internacional es la fuerza motriz de este proceso.

El informe no sólo justifica el financiamiento de CidA a las compañías mineras canadienses en sus actividades y operaciones en el extranjero, en términos del dogma neoliberal respecto al libre mercado, sino que asigna al sector privado la responsabilidad que la mayor parte de los teóricos internacionales del desarrollo (bajo el consenso post-Washington) asignan al Estado a al sector público, es decir, la de asegurar que el crecimiento económico sea socialmente incluyente, que las políticas «a favor del crecimiento» sean también «a favor de los pobres». De tal modo, CIDA asigna a una institución capitalista, la corporación minera, cuya única responsabilidad fiduciaria es generar ganancias para sus accionistas, actuar como agente de un desarrollo socialmente incluyente, resumiendo el pensamiento mágico de los diseñadores de políticas al servicio del capital. En este papel asignado por este informe al sector privado, el comité gobernante con mayoría del Partido Conservador propone reconfigurar el cida de mejor manera para servir a las corporaciones canadienses al salir al extranjero, comenzando por el sector minero. En relación con esto, el informe da prioridad a las sociedades públicas-privadas, como los controvertidos proyectos con las compañías mineras multimillonarias que ya están siendo piloteadas en países como Ghana, Burkina Faso y Perú como una «importante herramienta de la programación del desarrollo de [CIDA]» (House of Commons, 2012: 99).

Este informe del comité, opina la vocera de Mining Watch, Catherine Coumans, no sólo vincula la ayuda canadiense con los intereses mineros, «de hecho restructuraría a CIDA para servir mejor a los intereses del sector corporativo». ${ }^{12}$

${ }^{/ 12 /}$ Es evidente que ongs como Mining Watch, que son parte de una creciente sociedad civil internacional que apoya a comunidades afectadas negativamente por la minería, son muy críticas de la agenda 
No es que se requiera esta reestructuración. Como están las cosas, las organizaciones no gubernamentales (ongs) canadienses como Development and Peace y la organización religiosa KAIros han perdido, respectivamente, 50 y 100\% del financiamiento para sus proyectos de desarrollo precisamente por su apoyo activo a los grupos afectados negativamente por las operaciones mineras canadienses (Engler, 2012; Anónimo, 2012b; North, 2012). Por otra parte, las ongs, como World Vision, que apoyan la agenda del gobierno en apoyo del capital extractivo (incluyendo la pacificación de la resistencia proveniente de las comunidades afectadas), han recibido financiamiento adicional para mediar entre las compañías mineras, las comunidades afectadas negativamente y los gobiernos locales, cuyo apoyo es necesario para que a estas compañías se les conceda una «licencia social» para hacer avanzar estas operaciones. ${ }^{13}$

Además de las sociedades de CidA con las compañías mineras, el informe del comité recomienda a CiDA lo siguiente: 1) considere ofrecer préstamos y utilizar «otros instrumentos financieros» para apoyar a las corporaciones; 2) promover intercambios «revolventes entre CIDA y el sector privado para mejorar la capacidad de la agencia de servir a los intereses corporativos canadienses», y 3) «participar en el cambio de las instituciones, regulaciones, políticas y supervisión democrática en los países en desarrollo de modo que se promueva la inversión canadienses, particularmente en los países en desarrollo con sectores importantes en los recursos naturales» (MiningWatch, 2012, énfasis nuestro).

Lo que ignora el informe del comité de situación y lo que señala correctamente MiningWatch es que, durante la pasada década, Cida ya ha estado

del gobierno para CIDA. El «dinero para ayuda», señala Coumans (2012), «se supone que se dirija a la pobreza, no a promover los intereses comerciales de las compañías mineras canadienses». Ni tampoco, añade, «debería subsidiar las obligaciones de las compañías mineras para beneficiar a los residentes afectados y rehabilitar los ambientes perjudicados».

${ }^{13 /}$ Nótese, como ejemplo, el contrato concedido por ciDa a World Vision Canada, que recibió más de $\$ 89$ millones en ayuda extranjera en 2010 (después de Care Canada, con cerca de \$99 millones), para un proyecto con Barrick Gold en Perú (North, 2012; Engler, 2012). 
proveyendo apoyo técnico sobre la ley de minas y las reformas administrativas en varios otros países, repasando incluso la legislación relevante. Según Coumans (2012), la trayectoria de CIDA «ha consistido en debilitar los controles estatales, privatizar la industria del Estado y reducir las regalías, impuestos y otras medidas para captar beneficios económicos para atraer la inversión extranjera». Sobra decir que las acciones y políticas del gobierno a este respecto - y el informe del comité de situación - son completamente consistentes con una agenda imperialista para promover los intereses económicos de la clase capitalista, que inevitablemente se equiparan con el interés nacional.

\section{Costos y Beneficios Del CAPital EXTRACTIVo Y DEL IMPERIALISMO EXTRACTIVISTA}

Los devastadores impactos socioambientales de la industria de la extracción, especialmente la minería, en las economías y sociedades en el mundo son de conocimiento público, como lo son los conflictos políticos y sociales que han generado. ${ }^{14}$ No obstante, muchos analistas de las políticas y diseñadores de éstas en las democracias capitalistas avanzadas y la comunidad internacional del desarrollo asumen la visión de que estos riesgos y costos tienen que contrastarse con los beneficios potenciales del desarrollo de los recursos naturales. El argumento que proponen es que los proyectos de la industria extractiva basados en la IED generan empleos bien pagados y derivados o vínculos con actividades productivas (a favor del crecimiento), así como ofrecen recursos fiscales que pueden utilizarse para saldar las deudas del gobierno o ser movilizados (gastados o invertidos) para el beneficio público. Esta creencia es

\footnotetext{
/14/ Véase, respecto a estos conflictos ambientales a partir de la resistencia de las comunidades afectadas por las operaciones canadienses u otras mineras, entre otros, los numerosos reportes de Canada Mining Watch, la página de «Minas y comunidades» (〈http://www.minesandcommunities.org〉), Clark (2002) y North, Clark y Patroni (2006).
} 
compartida por muchos gobiernos, incluyendo a regímenes neoliberales «progresistas» en Sudamérica, orientados por el consenso post-Washington hacia la meta del crecimiento y el crecimiento incluyente. En el contexto de una fuerte demanda global continuada por energía, minerales industriales y metales, los productos agroalimentarios y otras mercancías, estos regímenes están comprometidos con la dependencia en la IED y el desarrollo de la industria de los recursos naturales, así como con las exportaciones de mercancías primarias. La diferencia respecto a años anteriores de experiencia con esta estrategia - una diferencia que se aplica incluso a regímenes abiertamente neoliberales como Colombia - consiste en que el «nuevo extractivismo» implica un régimen regulador y una preocupación por generar mejores contratos con las compañías mineras y captar una porción mayor del botín en forma de rentas por los recursos (regalías e impuestos).

Incluso un análisis sumario de la evidencia a partir de los lugares de extracción de recursos naturales en América Latina y el mundo muestra que son las comunidades más directamente afectadas por las actividades de extracción de recursos (exploración, perforación, cosecha, y en especial la minería y fractura) las que tienen que asumir los riesgos ${ }^{15}$ y soportar los costos directos e indirectos de estas actividades, incluyendo el daño a la salud, ambiente y formas de vida, al tiempo que reciben pocos de los beneficios - beneficios que, en su mayor parte, son exportados, con una porción relativamente pequeña dirigida al Estado-. En los Estados posneoliberales de Sudamérica, esta porción tiende a ser mayor, mientras que en las jurisdicciones sujetas a formas neoliberales de gobernanza (privatización frente a nacionalización, mercados desregulados, liberalización financiera y de productos) las rentas por los recursos que capta el gobierno como parte de las ganancias son mínimas, tan

/15/ Por supuesto que las compañías arriesgan las inversiones de sus accionistas, pero estos riesgos son escasos en comparación con los riesgos que tienen que asumir las comunidades afectadas por las actividades mineras ( $y$ con escaso o nulo poder para influir en la toma de decisiones y en las políticas en esta área). 
bajas como $1.2 \%$, en el caso de México. ${ }^{16}$ Dada la desigual distribución geográfica de los recursos naturales, las corporaciones mineras buscan invertir en el sector de la extracción en países donde podrían pagar mayores impuestos y regalías pero donde puedan obtener mayor rentabilidad con base en el volumen. Los factores operativos en las decisiones corporativas para invertir en la extracción de recursos parecen ser la apertura a la inversión extranjera, la tasa prevista de retorno del capital invertido y la seguridad legal que se concede a la propiedad privada y la repatriación de las ganancias. ${ }^{17}$

116/ En su más reciente reporte, el Auditor General de México, según el periodista de investigación Francisco López (2012), puso el dedo en la llaga que ha causado que México sangre minerales profusamente a lo largo de años. El auditor estableció que las cuotas que pagan las compañías mineras, 70\% de las cuales son canadienses, por sus concesiones para explotar los minerales están por debajo de los procedimientos administrativos. El reporte del auditor señala: «la cantidad de cuotas que se paga actualmente es simbólica y contrasta con los volúmenes extraídos de recursos minerales no renovables, dado que su valor está muy por encima de las cuotas de concesión que cobra el Estado, como observa en el periodo 2005 a 2010, cuando el valor de la producción alcanzó 552 mil millones de pesos [46 mil millones de dólares estadounidenses] y las cuotas que se cobran eran de apenas 6.5 mil millones de pesos [543.4 millones de dólares], cerca de 1.2\% de la primera». Además, señala, no es evidente que las cuotas pagadas fueran realmente pagadas, revelando la «laxitud extrema en la aplicación de la ley» y las «omisiones de las autoridades para vigilar el cumplimiento» de la ley (López, 2012: 31).

/17/ Entre las naciones en desarrollo, supuestamente «sólo la República Democrática de El Congo, Indonesia y Mongolia compiten con Brasil, Chile y Perú para obtener beneficios financieros de la explotación de sus arcas de tesoros minerales» (Reuters, 30 de agosto de 2011). Sin embargo, no parece existir un consenso entre los gobiernos de Sudamérica respecto a cómo enfrentar este desafío o cómo compartir las rentas de los recursos. Colombia es actualmente el destino preferido de los inversionistas extranjeros (seguido por Brasil y Chile, y luego por México); aplicó una política de «flexibilidad» para fijar las tasas por regalías (1\% en el caso de la minería de oro) y aún no ha decidido cómo o cuándo se aplicarán. Por otro lado, Chile todavía está «negociando» con las compañías mineras su sistema de impuestos «voluntarios», mientras que el nuevo gobierno de Perú todavía tiene que redactar una nueva legislación (aunque las compañías mineras que operan en el país supuestamente han mostrado su acuerdo en pagar regalías más altas para mejorar el actual sistema, es decir más que las tasas actuales de 1-3\% sobre las ventas). La posición del presidente de Ecuador, Rafael Correa, es más clara: aunque por el momento el gobierno no cobra regalías en absoluto por la minería - una tasa futura por regalías de 3-5\% y posiblemente un impuesto por ganancias súbitas están planeándose - las compañías petroleras tienen que pagar una tasa de regalías de 8\%, 
Consideremos el caso de Argentina, el sexto país en términos de minería en el mundo y, a diferencia de México, un ejemplo de un Estado posneoliberal comprometido con un modelo económico que promete captar una mayor porción de la riqueza de los recursos del país y una distribución más equitativa de esa riqueza. Para promover la minería, se exenta a las nuevas minas de todos los impuestos federales, provinciales y municipales por cinco años. Las corporaciones mineras transnacionales no pagaron impuesto alguno por sus exportaciones hasta 2008, no se les obliga a hacer depósitos de sus recibos de divisas extranjeras por exportaciones en el sistema financiero y no pagan impuestos sobre sus importaciones de maquinaria y equipo (Aldecoa, 2009: 4-5). Y aunque los Estados provinciales son técnicamente los propietarios de los recursos naturales, las corporaciones mineras no les pagan impuestos a ellos o a los municipios. Sólo siete de los 23 gobiernos provinciales cobran regalías por los minerales extraídos (Solanas, 2007bः 1). En aquellos casos en que se cobran regalías, el método consiste en calcular el valor de los minerales en el punto de extracción e imponer una regalía máxima de 3\% después de deducir los costos de transportación, refinería y fundición, otros costos de tratar los minerales y los costos de comercialización. Después de las deducciones, el gobierno recibe pagos por regalías de $1-1.5 \%$ del valor de los minerales seleccionados, el que se compara precisamente con la tasa del impuesto efectivo pagado por las compañías mineras canadienses en México.

Los críticos de la extracción de recursos naturales como modelo de desarrollo reconocen que los casos de México y Argentina - el primero un ejemplo de un régimen de políticas neoliberales, mientras que el segundo posee un

poniéndolo en el mismo camino que Evo Morales, quien, al asumir el poder del Estado, obligó a las compañías extranjeras en este sector a aceptar no sólo un incremento en impuestos y en la tasa de regalías por las extracción de recursos, sino también un impuesto por ganancias inesperadas de $70 \%$ (Raymond James, Mining Team, 2008). En cuanto a México, se ha señalado ya que los inversionistas extranjeros en el sector minero gozan de una tasa cero por regalías y una tasa efectiva de impuestos sobre las operaciones (sobre ventas más que ganancias) de 1.2\%. 
régimen posneoliberal- podrían ser extremos, pero ellos argumentan que develan una dinámica fundamental para el capitalismo extractivo y la explotación imperialista: el desarrollo desigual de las fuerzas productivas y una distribución burdamente desigual de la riqueza y el ingreso. Ya sea en el contexto de neoliberalismo o de un Estado posneoliberal, el saqueo de los recursos de un país (un rasgo básico del imperialismo en cualquier forma) deriva en la expatriación del capital extranjero de la mayor parte de los beneficios económicos bajo la guisa de ganancias sobre el capital invertido, con una porción relativamente limitada apropiada por el gobierno y escasos beneficios que fluyen a las comunidades locales, las que soportan la mayor carga de los costos sociales, económicos y ambientales asociados. La evidencia social científica disponible sobre este tema es voluminosa aunque controvertida.

Las operaciones del capital extractivo implican no sólo enormes costos sociales y ambientales, sino también serios costos económicos y políticos. La investigación (Petras y Veltmeyer, 2009), así como la evidencia anecdótica sugieren que, incluso en el caso de los regímenes posneoliberales o regulatorios nacionalistas - como los establecidos por Cristina Kirchner en Argentina, Luiz Inácio Da Silva (Lula) en Brasil y Evo Morales en Bolivia-, el gobierno tiende a tomar partido por las compañías mineras extranjeras en contra de las comunidades en cualquier conflicto (por recursos globales comunes de tierra, agua y subsuelo) que surgen de las operaciones de la industria extractiva y de la minería ${ }^{18}$ La teoría afirma que lograríamos explicar esta coincidencia de

/18/ «El caso más evidente de ello es el presidente Humala que, al llegar al cargo, cambió su tono de apoyo a las comunidades y nacionalista en recursos al tono que le tocaran las compañías que propusieron construir la mayor mina de cobre ( $y$ oro), prometiendo al país miles de millones de dólares en rentas por los recursos. Otro caso es el de Evo Morales en el controvertido proyecto TipNis del gobierno boliviano para construir una carretera que atravesara una reserva indígena protegida en contra de la vehemente oposición y resistencia organizada de las comunidades indígenas afectadas por el proyecto. Más recientemente, el presidente Correa en Ecuador ha convocado al apoyo de sus colegas presidentes en Perú y Colombia para lidiar con los “ambientalistas radicales" en su oposición a los planes de subastar 16 nuevos lotes de tierra en territorios indígenas para la exploración y la 
intereses económicos entre las compañías y los gobiernos en un régimen de extracción de recursos: ganancias para las primeras y rentas para los segundos. De tal forma, incluso en el caso del régimen híbrido de Ollanta Humala en Perú (una mezcla de nacionalismo de recursos, populismo y tecnocracia neoliberal), el gobierno sigue apoyando a las compañías mineras, como se refleja en la lucha que realizan las comunidades locales en la región de Cajamarca en contra del proyecto gigante de minería Newmont, Conga ${ }^{19}$ Aunque el gobierno accedió a ordenar una revisión independiente de los planes para la ampliación de la mina antes de realizarla, el mismo Humala dejó claro que el país no podría permitirse detener el proyecto de 4.8 miles de millones de dólares - y no lo haría, independientemente de la revisión-.

\section{DiNÁMICA DEL CONFLICTO POR LOS RECURSOS Y}

LA LUCHA EN CONTRA DE LA MINERÍA

Los agentes de la resistencia en contra de las incursiones imperiales del capital en la explotación de los recursos naturales - al menos en el contexto latinoamericano - son las comunidades predominantemente indígenas que pueblan las áreas concesionadas por los diferentes gobiernos a las compañías

perforación petrolera (Fraser, 2012) - y la oposición al desarrollo de la "buena minería” (necesaria para que el país "escape de la pobreza”). La solución, enfatizó Correa, no consiste en oponerse a la minería por sí misma, sino a la mala minería: "no podemos ser pordioseros sentados en un saco de oro"» (ANDINA, 2012).

/19/ La mina de Conga, un proyecto conjunto entre la compañía con sede en Denver, Newmont Mining Corp., Buenaventura de Perú y el Banco Mundial (por medio de la International Financial Corporation), ayudaría a la compañía (y al gobierno) a alcanzar la meta de producir 7 millones de onzas de oro y 400 millones de libras de cobre para 2017 — un importante impulso tanto al PIB como a las rentas (regalías e impuestos) recolectados por el gobierno y las ganancias de la compañía-. Pero estas inmensas rentas y ganancias se darían a costa de la devastación de la tierra, el agua y el sustento de las comunidades indígenas locales que rodean las operaciones mineras. 
mineras extranjeras para su exploración y explotación ${ }^{20}$ Pero los agentes también incluyen a una diversidad de grupos de la sociedad civil y ongs que han sido arrastrados al conflicto entre el capital global y las comunidades locales. ${ }^{21}$ Y las fuerzas de resistencia al imperialismo de los recursos incluyen a nuevos movimientos sociales formados para protestar por el daño al ambiente, la salud y las formas de sustento de la población local, y los mineros mismos que enfrentan condiciones de trabajo desde insalubres hasta las que ponen en riesgo la vida. En otras palabras, muchos de estos movimientos están arraigados en aquellos «afectados» por los impactos de las operaciones de extracción de recursos y mineras - por ejemplo, la Red Mexicana de Afectados por la Minería (REM) y la Confederación Nacional de Comunidades del Perú Afectadas por la Minería (Conacami) - .

Según un foro de pueblos, comunidades y grupos afectados por las operaciones del capital minero y la industria de la extracción de recursos (Foro de los Pueblos Indígenas Minería, Cambio Climático y Buen Vivir), reunido en Lima en noviembre de 2010, la explotación de los recursos minerales de América Latina en 2009 había alcanzado niveles hasta entonces jamás experimentados. De especial preocupación fue la región del Amazonas, cuyos abundantes depósitos de oro, bauxita, piedras preciosas, manganeso y uranio son buscados por las compañías multinacionales que operan en el sector minero. Otra preocupación fue la asociación percibida entre las corporaciones multinacionales en el sector y un conjunto de fundaciones y ongs. Al respecto, Eddy Gómez Abreu, presidente

/20/ Por lo general, los gobiernos reconocen los derechos y la propiedad de la tierra en las áreas pobladas por las comunidades indígenas y también el derecho de las comunidades indígenas a ser consultadas respecto a las operaciones mineras propuestas y otros proyectos de extracción en su territorio. Sin embargo, es común que los gobiernos se reservan a concesionar a las compañías mineras el derecho a explotar los recursos subterráneos bajo una «licencia social» concedida bajo un régimen regulatorio (protección ambiental) y un acuerdo de pago de regalías o impuestos.

121/ Un ejemplo de una de estas ongs es Global Response, que ha preparado un manual que puede utilizarse por los activistas para lidiar y contraponerse con las diversas estrategias operadas por las compañías mineras para superar la resistencia local. Véase Zorrilla (2009). 
del Parlamento Amazónico Internacional, declaró que la organización contaba con «evidencia incontrovertible de que estas transnacionales y fundaciones, bajo la cobertura de supuestas preocupaciones ecológicas, religiosas o humanitarias, han colaborado en el esfuerzo por extraer diamantes, minerales estratégicos y en el espionaje genético, así como en experimentos médicos ilegales en la población indígena» (Sena-Fobomade, 2011). Efectivamente, se argumentaba que una de las tácticas de las compañías mineras era utilizar a las fundaciones y otras oNGs como frentes de relaciones públicas y como organizaciones de compilación de datos de inteligencia, para asegurar el consentimiento de la población local con sus proyectos. De ser cierto, estas fundaciones y ongs se remontan a la sórdida historia de los misioneros europeos como punta de lanza para la expropiación de las tierras aborígenes en las Américas.

En cuanto a la correlación de fuerzas involucradas en este conflicto por los recursos, hay varios temas implicados, incluyendo el de las tácticas. Por un lado, las fuerzas de la resistencia utilizan tácticas como marchas y manifestaciones, bloqueos en carreteras e ingresos y otras formas de acción colectiva directa, para interferir con las operaciones mineras (Zorilla, 2009). Por otro lado, las tácticas de las compañías mineras incluyen visitar la comunidad para recabar información y evaluar la situación local (el grado de oposición, identificar a los líderes a ser cooptados o «neutralizado») bajo falsos pretextos, como presentarse como miembros de una ONG preocupada por el bienestar de los indígenas; organizar reuniones públicas con ayuda de sus aliados locales o funcionarios amigables; sobornar a los funcionarios de gobierno con la promesa de empleos y fondos para desarrollo social; ${ }^{22}$ manufacturar una «licencia

122/ Un ejemplo de esta táctica es la aproximación utilizada por Goldcorp, la firma canadiense que domina la industria mundial global de extracción de oro, para consolidar su operación en Zacatecas, el mayor productor de oro y plata del país. Con el propósito declarado de ayudar al estado a promover el desarrollo social de las comunidades que sufren de un alto grado de marginación, Goldcorp anunció en Zacatecas una bolsa de 72 millones de pesos (Anónimo, La Jornada Zacatecas, 15 de marzo de 2012), además de los 436 millones que gastó en ese estado en 2011. 
social» al negociar con un grupo local que apoye el proyecto (este apoyo obtenido usualmente por medio de financiamiento indirecto); ofrecer empleo con altos ingresos a los miembros desempleados de la comunidad o trabajar con los contratistas locales; comprar tierras con acceso a las concesiones; infiltrar la oposición para sembrar la división y la desconfianza; los litigios estratégicos contra la participación pública, falsas acusaciones, intimidación y amenazas de muerte, e incluso acción paramilitar y asesinatos. En última instancia, las compañías mineras acuden a la violencia directa y a la coerción de las fuerzas militares, paramilitares o policiacas para vencer la oposición a sus operaciones mineras altamente lucrativas.

Desde la perspectiva del capital extractivo y los gobiernos locales (el Estado neoliberal o posneoliberal), estas tácticas reflejan una preocupación por proteger las extraordinarias tasas de ganancia asociadas con las operaciones extractivas, así como las rentas por los recursos que persiguen los gobiernos para financiar programas de desarrollo. Desde la perspectiva de las comunidades locales, sin embargo, no sólo está en juego la salud de sus miembros y el control soberano de sus territorios étnicos o nacionales, sino el ambiente del cual dependen su sustento y su forma de vida, y sin duda la vida misma. A este respecto, Gómez Abreu informó, en un análisis socio-metabólico de la economía, más de un millón de personas en la cuenca del Amazonas sufre de enfermedades derivadas de la exposición o ingestión de substancias tóxicas y carcinógenas, como el mercurio de las operaciones mineras. En el mismo sentido, el investigador Edgardo Alarcón documentó evidencias de que la ciudad peruana de Oroya es una de las diez ciudades más contaminadas del mundo, con altos niveles de plomo y sulfuro en el aire y altos niveles de carcinógenos relacionados con la minería como el cadmio, arsénico y antimonio en el suelo, productos agrícolas alimenticios y en el suministro de agua; estas toxinas también se han detectado en otros pueblos y comunidades de los alrededores (Sena-Fobomade, 2011).

Una de las principales organizaciones que participaron en el Foro de Lima en 2010, Conacami, denunció el hecho de que para fines de 2010 la 
gran mayoría de los sitios ancestrales en su territorio estaba en manos de las compañías mineras y petroleras (las «transnacionales») y que hasta $72 \% \mathrm{del}$ territorio nacional de Perú había sido concesionado para el propósito de exploración y explotación de los recursos naturales del país (Lust, 2012). Al respecto, Conacami alertó a los participantes del foro acerca de las acciones del gobierno - en ese momento bajo el control del predecesor neoliberal de $\mathrm{Hu}$ mala, el presidente Alan García - al anunciar que 33 mega proyectos eran de «interés nacional»y, por ende, según Sana-Fobomade, retirando la necesidad de que las compañías sometieran estudios de impacto ambiental. ${ }^{23}$

Éstos y otros informes reflejan el hecho de que Perú, junto con Ecuador, es uno de los principales lugares de América Latina para la lucha de clases a través de la extracción y explotación de los recursos naturales. Un punto focal importante en esta lucha sigue siendo la mina Conga, la mayor operación de extracción en Perú y uno de los más grandes de todo el hemisferio. En el momento de escribir estas líneas, el proyecto Conga está «en espera», se estancó como consecuencia de la resistencia organizada de las comunidades locales y el gobierno regional de la región de Cajamarca.

En febrero de 2012, en la época en que la presidenta argentina Cristina Fernández confrontaba una situación similar, la resistencia reinante al proyecto Conga asumió la forma de una marcha nacional «a favor del agua y la vida», una movilización que logró un amplio apoyo del público, así como la participación activa de diversos grupos y sectores sociales. Las comunidades locales —en su lucha en contra del capital minero y la industria de la extracción iban a tener éxito para detener un proyecto que el gobierno había declarado de importancia estratégica- habrían de dar un impulso considerable a las fuerzas de la resis-

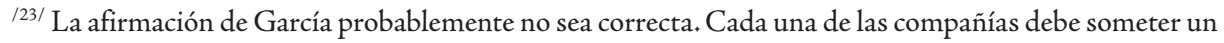
reporte ambiental por cada proyecto propuesto, independientemente de si se le considera de «interés nacional». El punto es, señala Lust (2012), que estas revisiones «no juegan papel alguno en la política y en la toma de decisiones del gobierno respecto a la aprobación o no del proyecto o concesión para explorar y extraer». 
tencia en toda la región. Esa victoria a favor «del pueblo» —una de las pocas en años recientes - asentaría un cambio en las fuerzas de clase. Al mismo tiempo, lo que está en juego es muy valioso, tanto para las compañías mineras como para el Estado (tanto en Perú como en otras partes de la región). Es probable que el gobierno apoye al capital minero y actúe para generar las condiciones que permitan que el proyecto continúe, ya sea a través de la cooptación o de la represión. Desafortunadamente, esta historia se repite en diversos escenarios de conflicto en la región y fuera de ella. Las dinámicas precisas de esta lucha y el resultado probable son inciertos y son blancos de mayor estudio.

\section{Conclusión}

La pregunta central abordada en este artículo era la de si el nuevo extractivismo representa un nuevo modelo de desarrollo para América Latina o, como argumentan algunos, una nueva forma de imperialismo - la forma que el capitalismo y el imperialismo están tomando en el siglo xxI-. Nuestra respuesta a esta pregunta puede sintetizarse sucintamente en la noción de «Canadá imperial». El centro canadiense para el estudio del conflicto por los recursos (Canadian Centre for the Study of Resource Conflict) comisionó y publicó en 2009 un estudio intitulado «Responsabilidad social corporativa y antecedentes para las firmas canadienses de minería y exploración en el mundo en desarrollo» (Corporate Social Responsibility and Footprints for Canadian Mining and Exploration Firms in the Developing World) que afirmaba que «las compañías canadienses han constituido el grupo más importante involucrado en incidentes desafortunados en el mundo en desarrollo». Eso es expresarlo eufemísticamente. La revisión que hemos presentado de la economía política de la extracción de recursos naturales en América Latina sugiere un sistema en crisis, una crisis que ha dado lugar a diversas fuerzas de resistencia y un cambio en la correlación de fuerzas, lo que anticipa una prolongada lucha en contra del capitalismo y el imperialismo de extracción. Está en juego en esta 
lucha el posterior desarrollo de las fuerzas productivas mundiales basadas en la extracción de materias primas y recursos naturales estratégicos que amenazan con encerrar lo que queda de los territorios comunales en el mundo y a la vez privatizar $y$ «mercantilizar» estos recursos, arriesgando los sustentos y las comunidades de millones de personas en el sector popular de la sociedad. Lo que está en juego no podría ser más valioso: el bienestar de las personas en el mundo en posibilidad de vivir en solidaridad con los otros y en armonía con la naturaleza, en última instancia, la vida misma.

\section{REFERENCIAS}

Aldecoa, José (2009), «La gran mentira de las exportaciones mineras de Argentina», El Malvinense, 14 de abril, 1-5, en 〈http://www.malvinense. com.ar/snacional/0109/1003.htm >.

Andina (2012), «Presidente Correa plantea abordar problema de radicales antimineros», 30 de noviembre, en 〈http://www.noalamina.org/mineria-latinoamerica/mineria-ecuador/presidente-correa-planteaabordar-problema-de-qradicales-antiminerosq $>$.

Anónimo (15 de marzo de 2012), «Dona la empresa minera Gold Corp $\$ 72$ millones a Zacatecas», La Jornada Zacatecas, en 〈http://issuu.com/lajornadazacatecas.com.mx/docs/local15032012op».

Anónimo (2012a), «Sepa cómo es el mapa del territorio argentino en manos de extranjeros», laangosturadigital.com, en 〈http://www.laangosturadigital.com.ar/v3.1/home/interna.php?id_not=23416andori=web .

Anónimo (2012b), «Poor Mining Companies? Parliamentary Committee Report Calls for Cida Giveaway to Canadian Corporations», Mining Watch Canada, 8 de noviembre 8, en 〈http://www.miningwatch.ca/es/ node/7043>.

Arbix, Glauco y Scott Martin (2010), «Beyond Developmentalism and Market Fundamentalism in Brazil: Inclusionary State Activism without Sta- 
tism», ponencia presentada en el taller «States, Development and Global Governance», University of Wisconsin-Madison, Marzo 12-13.

Arellano, Juan Martin (2010), «Canadian Foreign Direct Investment in

Latin America», ponencia preparada para Dialogue on Canada-Latin American Economic Relations, mayo, Ottawa, North-South Institute. Au tr, Richard (1993), Sustaining Development in Mineral Economies: The Resource Curse Thesis, Londres, Routledge.

Auty, Richard (editor) (2001), Resource Abundance and Economic Development, Oxford University Press, en 〈http://www.wider.unu.edu/ research/1998-1999-4.2.publications.htm >.

Banco Mundial (2005), Extractive Industries and Sustainable Development. An Evaluation of World Bank Group Experience, Washington, World Bank, IFC, MIGA.

(2010), Rising Global Interest in Farmland: Can it Yield Sustainable and Equitable Benefits?, Washington, World Bank.

(2011), The World Bank Group in Extractive Industries. 2011 Annual Review, Washington, World Bank.

Bannon, Ian y Paul Collier (editores) (2003), Natural Resources and Violent Conflict: Options and Actions, Washington, World Bank.

Bebbington, Anthony (editor) (2011), Minería, movimientos sociales y respuestas campesinas. Una ecología política de transformaciones territoriales, Lima, Instituto de Estudios Peruanos, Centro Peruano de Estudios Sociales.

Bebbington, Anthony, Leonith Hinojosa, Denise Humphreys, Maria Burneo y Ximena Warnaars (2009), «Contienda y Ambigüedad: Minería y Posibilidades de Desarrollo», Debate Agrario, número 44.

Borras Jr., Saturnino, Jennifer Franco, Sergio Gómez, Cristóbal Kay y Max Spoor (2012), «Land Grabbing in Latin America and the Caribbean», Journal of Peasant Studies, volumen 39, números 3-4.

Bresser-Pereira, Luiz Carlos (2007), «Estado y mercado en el nuevo desarrollismo», Nueva Sociedad, número 210. 
Bresser-Pereira, Luiz Carlos (2009), Developing Brazil. Overcoming the Failure of the Washington Consensus, Boulder, Lynne Rienner Publications. Cepal (Comisión Económica para América Latina y el Caribe) (2009), Foreign Investment in Latin America and the Caribbean, Santiago, CEPAL. (2010), Time for Equality: Closing Gaps, Opening Trails, Santiago, CePal. (2007), Foreign Investment in Latin America and the Caribbean, Santiago, CEPAL.

Clark, Tim (2002), «Canadian Mining Companies in Latin America: Community Rights and Corporate Responsibility, Conference Report to Cerlac y Mining Watch Canada», Toronto, 9-11 de mayo.

Collier, Paul (2003), «Natural Resources, Development and Conflict: Channels of Causation and Policy Interventions», ponencia presentada en Annual World Bank Converence on Development Economics-Europe, 28 de abril, Washington, World Bank.

Collier, Paul y Venables, Anthony (2011), Plundered Nations? Successes and Failures in Natural Resource Extraction, Londres, Palgrave Macmillan.

Coumans, Catherine (2012), «Placer Dome Case Study: Marcopper Min», Marinduque, Philippines, en 〈www.miningwatch.ca/placer-domecase-study-marcopper-mine-marinduque-philippines $>$.

Cypher, James (2010), «South America's Commodities Boom. Developmental Opportunity or Path Dependent Reversion?», Canadian Journal of Development Studies, volumen 30, números 3-4.

De Echave, José (2008), Diez años de minería en el Perú, Lima, Cooperación.

Deininger, Klaus y Derek Byerlee (2011), Rising Global Interest in Farmland:

Can it Yield Sustainable and Equitable Benefits?, Washington, World Bank. Deneault, Alain y William Sacher (2012), Imperial Canada Inc: Legal Haven of Choice for the World's Mining Industries, Vancouver, Talonbooks.

Engler, Yves (2012), The Ugly Canadian: Stephen Harper's Foreign Policy, Vancouver, Halifax, ReD Publishing, Fernwood Publishing.

Fraser, Barbara (2012), «Oil and Gas Drilling on the Rise in Ecuador and Peru; Indigenous Communities Share Concerns», 10 de diciembre, en 
〈http://indiancountrytodaymedianetwork.com/article/oil-and-gasdrilling-rise-ecuador-and-peru-indigenous-communities-shareconcerns-146201>.

Girvan, Norman (2012), «Comments on "Introduction to the New Extractivism: A Collaborative Research Project"».

Gudynas, Eduardo (2010), «The New Extractivism in South America: Ten Urgent Theses about Extractivism in Relation to Current South American Progressivism», Bank Information Center, en 〈http://www.bicusa. org/en/Article.11769.aspx> (consultado el 14 de octubre de 2010). (2011a), «La izquierda de los límites al nuevo extractivismo», La Primera, Lima, 11 de mayo, en 〈http://www.diariolaprimeraperu.com/ online/columnistas/la-izquierda-de-los-limites-al-nuevo-extractivismo_85841.html〉.

(2011b), «Si eres tan progresista ¿Por qué destruyes la naturaleza? Neoextractivismo, izquierda y alternativas», Ecuador Debate, abril.

Haber, Stephen y Víctor Menaldo (2012), «Natural Resources in Latin America: Neither Curse Nor Blessing», ssRn Working Paper, Oxford Handbook of Latin American Political Economy, en 〈http://ssrn.com/ abstract $=1625504$ >

Harvey, David (2003), The New Imperialism, Oxford, Oxford University Press. House of Commons (2012), «Driving Inclusive Economic Growth: The Role of the Private Sector in International Development», Report of the Standing Committee on Foreign Affairs and International Development, November, Ottawa, Public Works and Government Services Canada.

Infante, Ricardo y Osvaldo Súnkel (2009), «Chile: hacia un desarrollo inclusivo», Revista CEPAL, volumen 10, número 97.

KaY, Cristóbal y Jennifer Franco (2012), The Global Water Grab: A Primer, Amsterdam, Transnational Institute.

Keenan, Karyn (2010), «Canadian Mining. Still Unaccountable», NAcla Report on the Americas, mayo/junio, en 〈https;//nacla.org/article/canadian-mining-still-unaccountablè. 
Lievesley, Geraldine y Steve Ludlam (editores) (2009), Reclaiming Latin America: Experiments in Radical Social democracy, Londres, Zed.

López, Francisco (2012), «Detener el saqueo minero en México», La Jornada, 28 de febrero.

López, Francisco y Mayra Eslava (2011), El mineral o la vida la legislación minera en México, México, COAPI.

Lust, Jan (2012), «Peru: Extractive Capital and Social Resistance», en James Petras y Henry Veltmeyer (editores), The New Extractivism in Latin America: A New Development Model or Imperialism of the 21st Century?, Zed Books Ltd.

Ministerio de Minas y Energía, República de Colombia (2012), Colombia Minera, Desarrollo Responsable, Bogotá, República de Colombia.

NorTh, Liisa (2012), «Possibilities of redress for harms from mining: Communities vs, corporations in Latin America», public talk, IDs Visiting Speakers Program, November 9, Halifax, Saint Mary's University.

North, Liisa, Timothy David Clark y Viviana Patroni (editores) (2006), Community Rights and Corporate Responsibility: Canadian Mining and Oil Companies in Latin America, Toronto, Between the Lines.

Paley, Dawn y Sakura Saunder (2012), Investing in Conflict-Public Money, Private Gain: Goldcorp in the Americas, Washington, Rights Action.

Panfichi, Aldo y Omar Coronel (2011), «Los conflictos hídricos en el Perú 2006-2010: una lectura panorámica», en R. Boelens, L. Cremers y M. Zwarteveen (editores), Justicia Hídrica. Acumulación, conflicto y acción social, Lima, Justicia Hídrica, Instituto de Estudios Peruanos, Fondo Cultural PUCP.

Petras, James y Henry Veltmeyer (2005). Empire with Imperialism: The Globalizing Dynamics of Neo-liberal Capitalism, Londres, Zed. (2009), What's Left in Latin America, Londres, Ashgate.

Raymond James Mining Team (2008), «Mining in la: Navigating the LA Landscape», RJ Equity Research, 12 de agosto, en 〈https://www.raymondjames.ca/rjl_marketing/dotca/en_CA/Equity\%20Capital\%20 
Markets/Equity\%20Research/Sample\%20Research/Sample\%20Research\%20pDFs/MiningLatinAmerica081208.pdf.

Reuters (30 de agosto de 2011), «Royalty debates revisit Latin American states», MAC: Mines and Communities, en 〈http://www.minesandcommunities.org .

SAcHs, Jeffrey y Andrew Warner (2001), «The Curse of Natural Resources», European Economic Review, número 45.

Sena-Foвomade (2011), «Se intensifica el extractivismo minero en América Latina», Foro Boliviano sobre Medio Ambiente y Desarrollo, en «http:// fobomade.org.bo/art-1109).

Solanas, Fernando (2007a), «El despojo de los metales argentinos (parte Iv)», Rebelión, 31 de julio, en 〈http://www.rebelion.org/seccion.php $? \mathrm{id}=9$,

(2007b), «El despojo de los metales argentinos (parte III)», Argenpress, 6 de agosto, en http://www.argenpress.info/notaprint.asp?num $=04$ 4436andparte $=3$ > .

Sosa, Milagros y Margreet Zwarteveen (2012), «Exploring the Politics of Water Grabbing: The case of large mining operations in the Peruvian Andes», Water Alternatives, volumen 5, número 2.

Stijns, Jean-Philippe (2006), «Natural Resource Abundance and Human Capital Accumulation», World Development, volumen 34, número 6.

Veltmeyer, Henry y Darcy Tetreault (2012), Poverty in Latin America: Public Policies and Development Pathways, West Hartford, Kumarian Press. White, Ben, Saturnino Borras, Ruth Hall, Ian Scoones y Wendy Wolford (2012), «The New Enclosures: Critical perspectives on Corporate Land Deals», Journal of Peasant Studies, volumen 39, números 3-4.

Zorrilla, Carlos (2009), Protegiendo a su comunidad contra las empresas mineras y otras industrias extractivas, Boulder co, Global Response. 
